\title{
Supplementary data
}

For

\section{Graphene@metal nanocomposites by solution combustion synthesis}

Alexander Khort ${ }^{i \dagger}+$, Valentin Romanovskii ${ }^{\$ \ddagger}$, Vasilina Lapitskaya ${ }^{\dagger}$, Tatyana Kuznetsova ${ }^{\dagger}$, Khabib Yusupov $^{\S}$, Dmitry Moskovskikh*, Yulyan Haiduk", Kirill Podbolotov ${ }^{\perp}$

${ }^{i}$ KTH Royal Institute of Technology, Stockholm 100-44, Sweden

${ }^{\dagger}$ A.V. Luikov Heat and Mass Transfer Institute of the National Academy of Sciences of Belarus, Minsk 220072, Belarus

\$Institute of General and Inorganic Chemistry, National Academy of Sciences of Belarus, Minsk 220072, Belarus

†National University of Science and Technology "MISIS", Moscow 119049, Russia

${ }^{\S}$ Lulea University of Technology, Lulea 971 87, Sweden

"Belarusian State University, Minsk 220030, Belarus

${ }^{\perp}$ Physical-Technical Institute of the National Academy of Sciences of Belarus, Minsk 220141 Belarus

*E-mail: khort@kth.se 
Figure S1 shows differences in the combustion process of reacting systems with low (1.75) and high (5) $\varphi$ values.

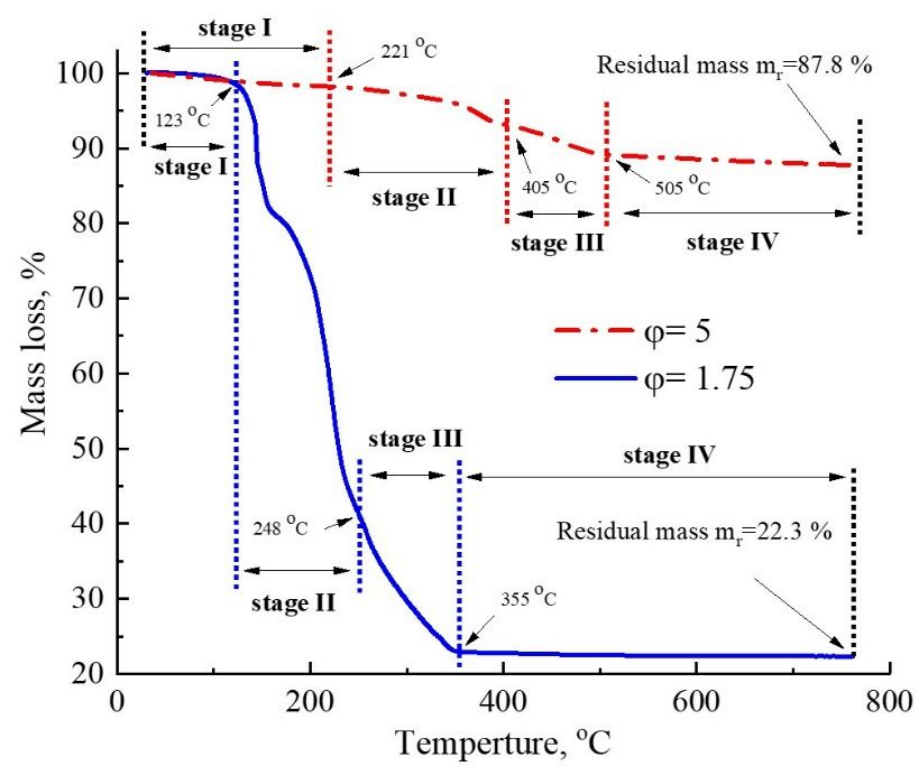

Figure S1. TG analysis of SCS process of G@CuNi samples with $\varphi=1.75$ and 5

The Raman spectrum mapping data of all the samples are shown in figs. S2-S4.

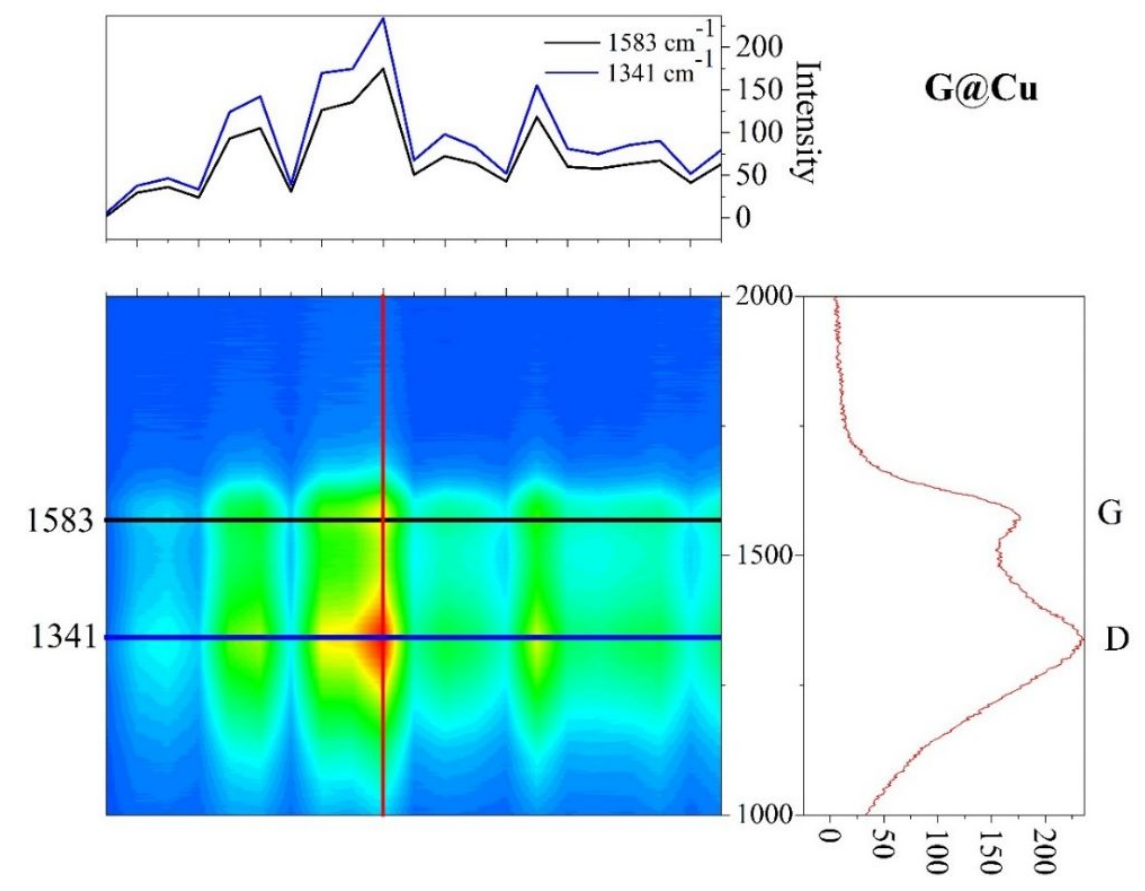

Figure S2. Raman mapping of G@Cu powder, also showing intensities of G and D bands across scanning area (top insert) and single Raman spectrum (right insert) 

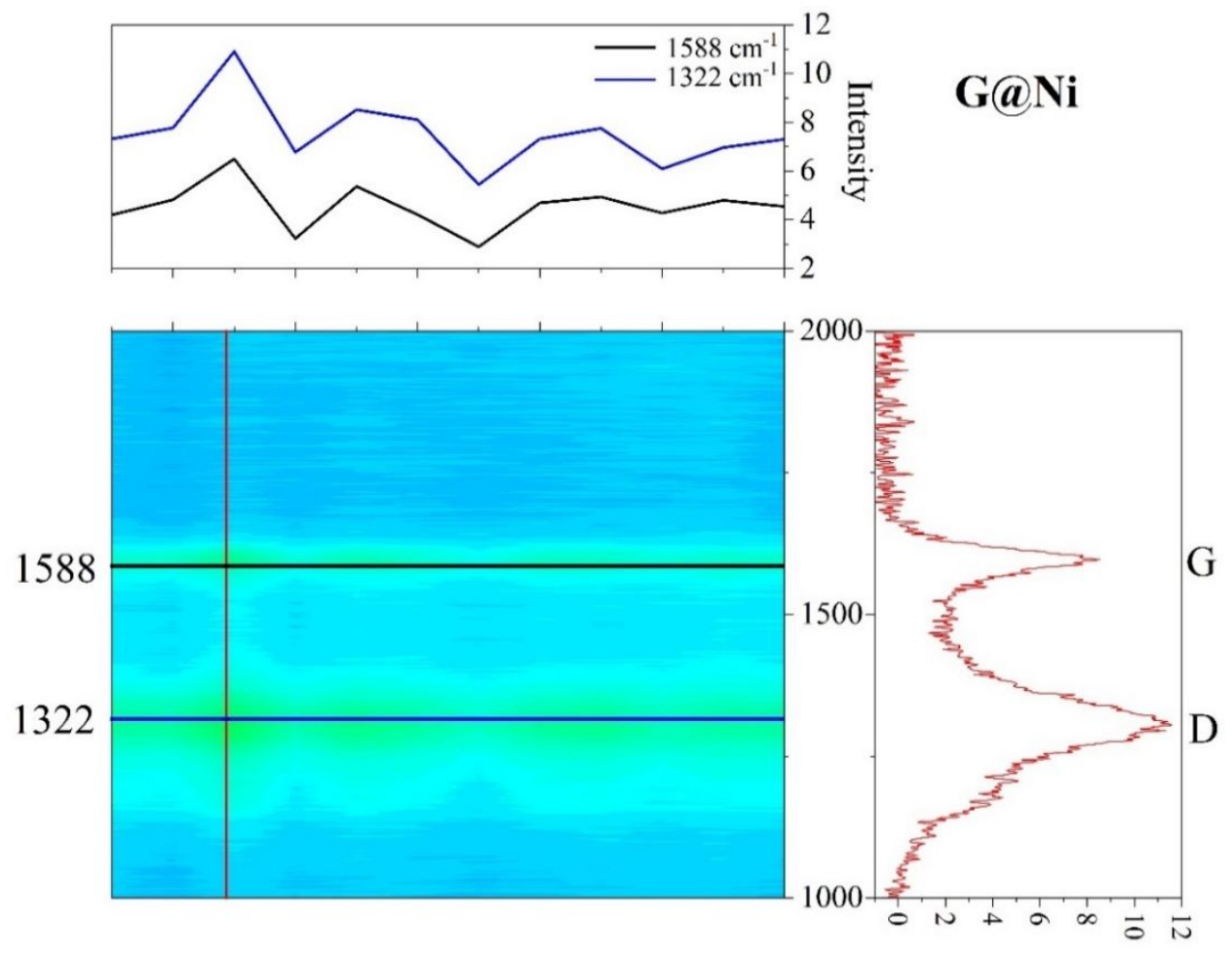

Figure S3. Raman mapping of G@Ni powder, also showing intensities of G and D bands across scanning area (top insert) and single Raman spectrum (right insert)

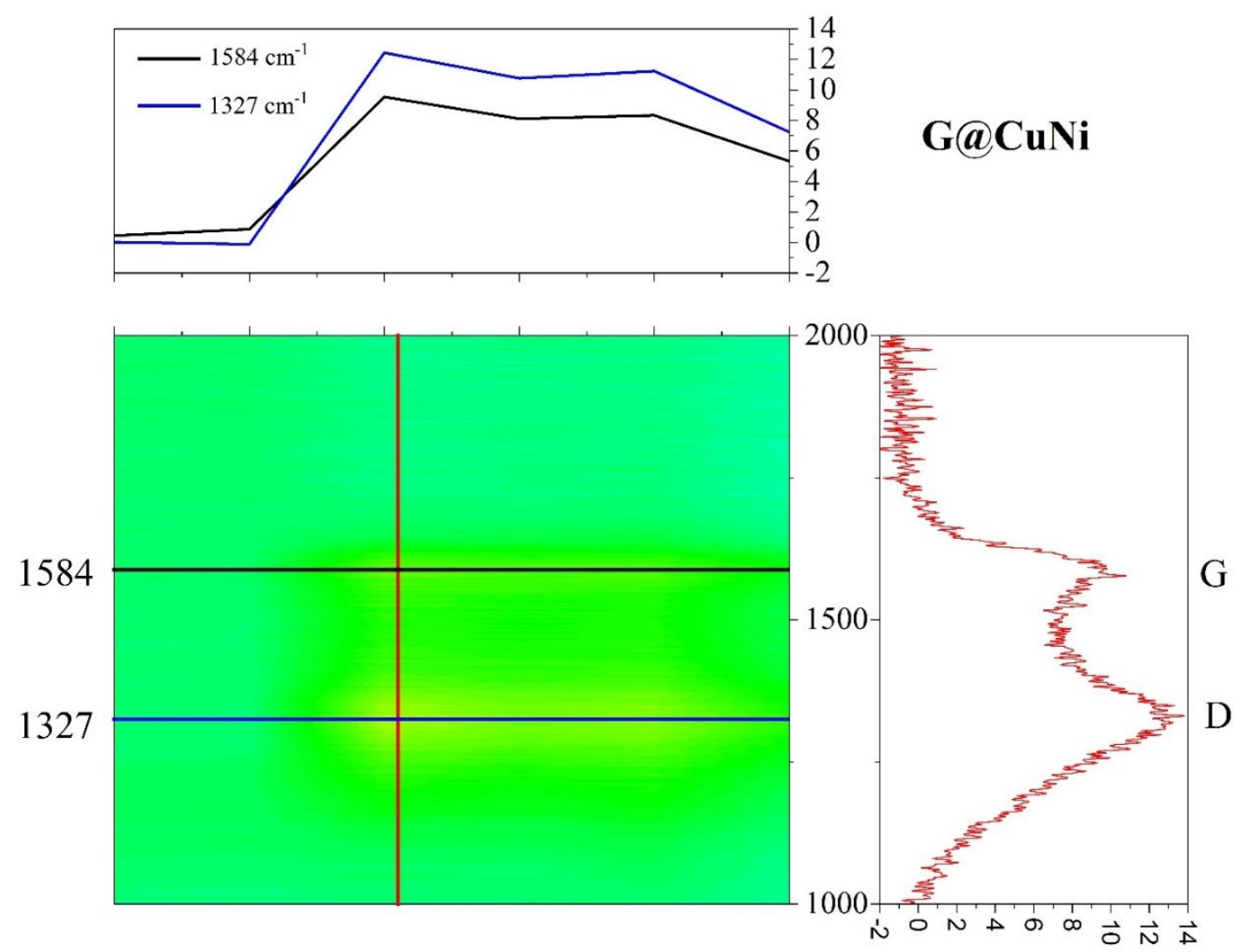

Figure S4. Raman mapping of G@CuNi powder, also showing intensities of G and D bands across scanning area (top insert) and single Raman spectrum (right insert) 
The Additional TEM images of G@Cu and G@Ni samples are sown in fig. S5.
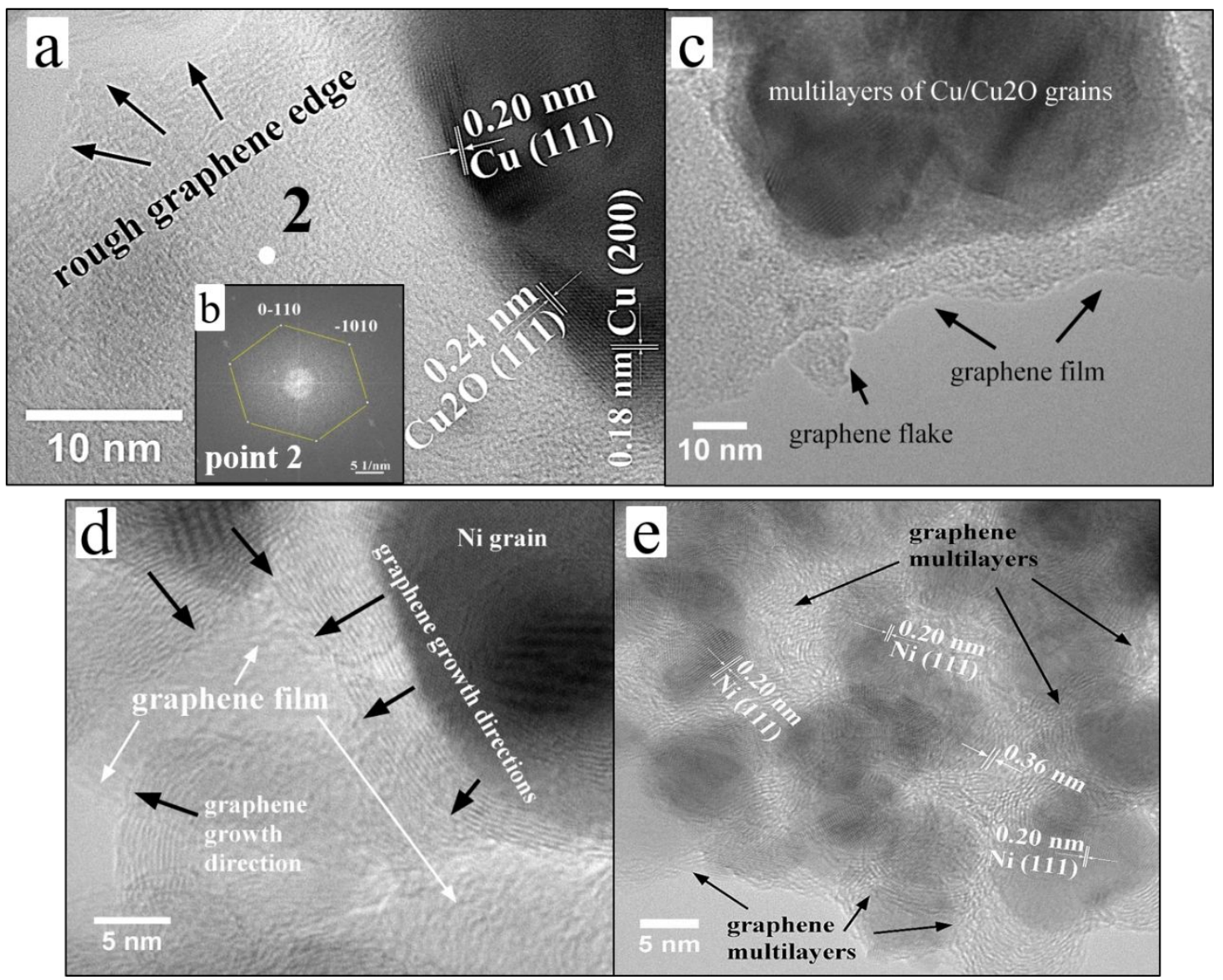

Figure S5. TEM images of (a-c) G@Cu and (d,e) G@Ni samples

The AFM images of all experimental samples are sown in figs. S5-S7. 

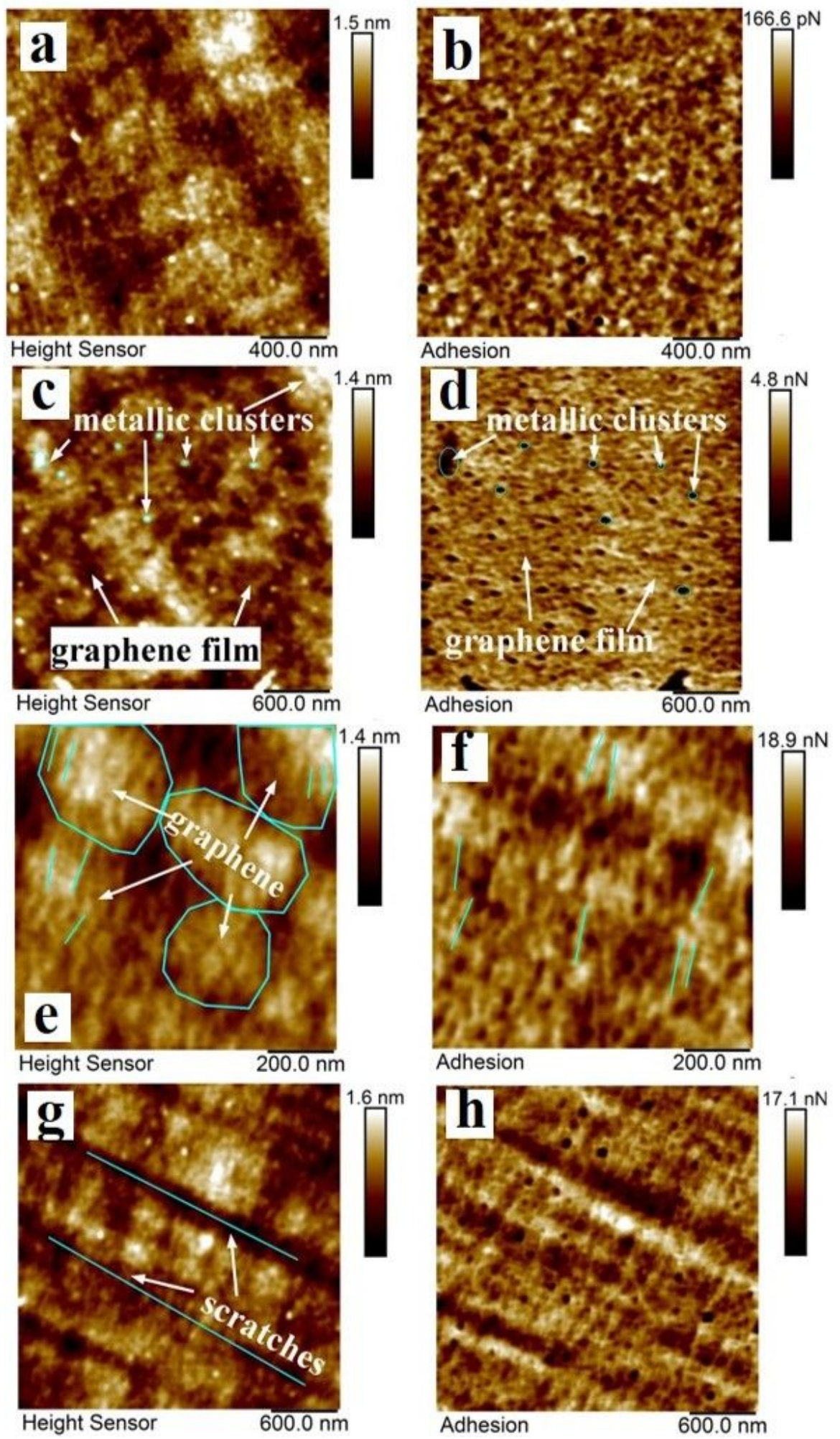

Figure S6. AFM images of G@Cu etched samples in (a, c, e, and g) topography and (b, d, $\mathrm{f}$, and $\mathrm{h}$ ) adhesion force modes 

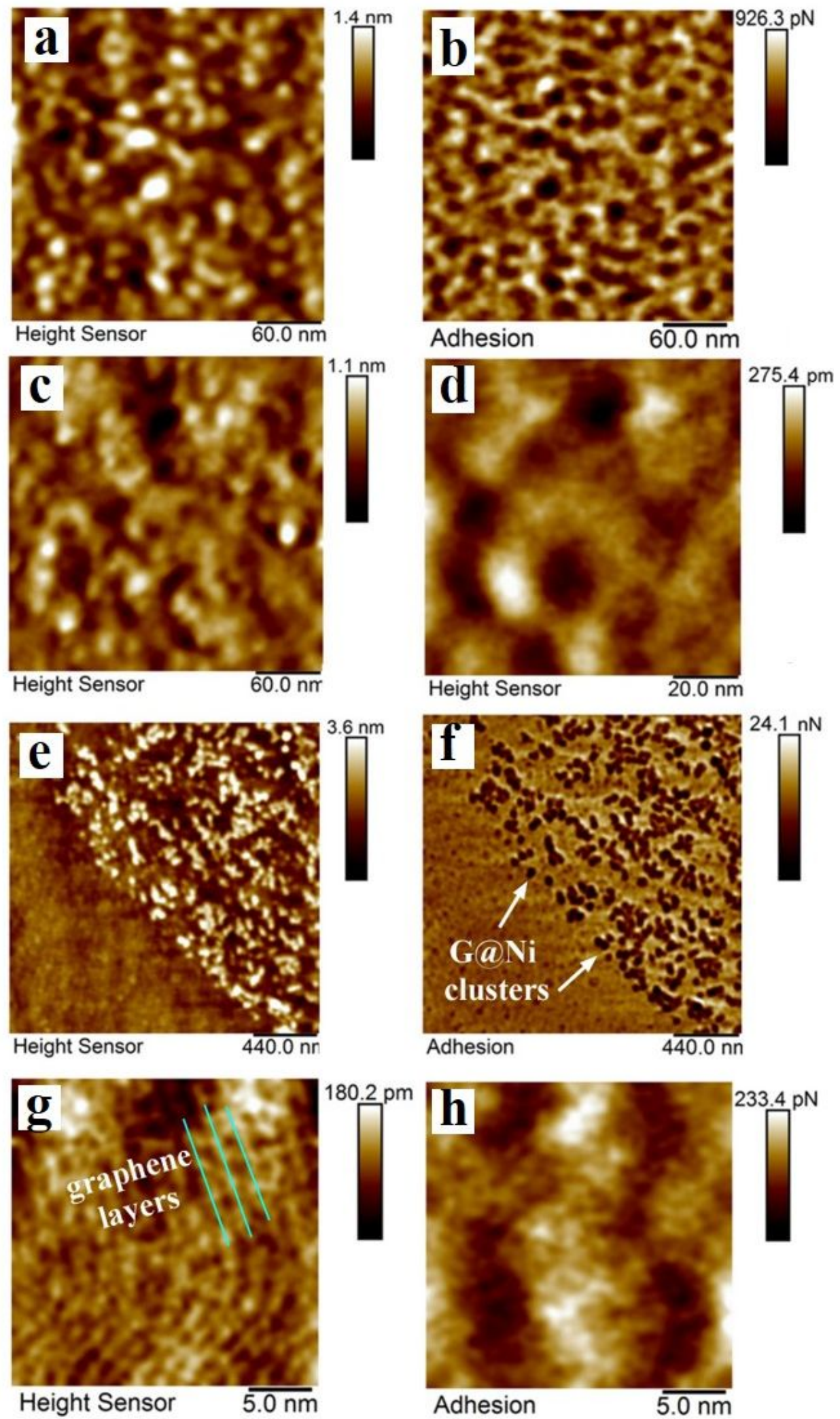

Figure S7. AFM images of G@Ni etched samples in (a, c, d, e, and g) topography and (b, f, and $\mathrm{h}$ ) adhesion force modes 

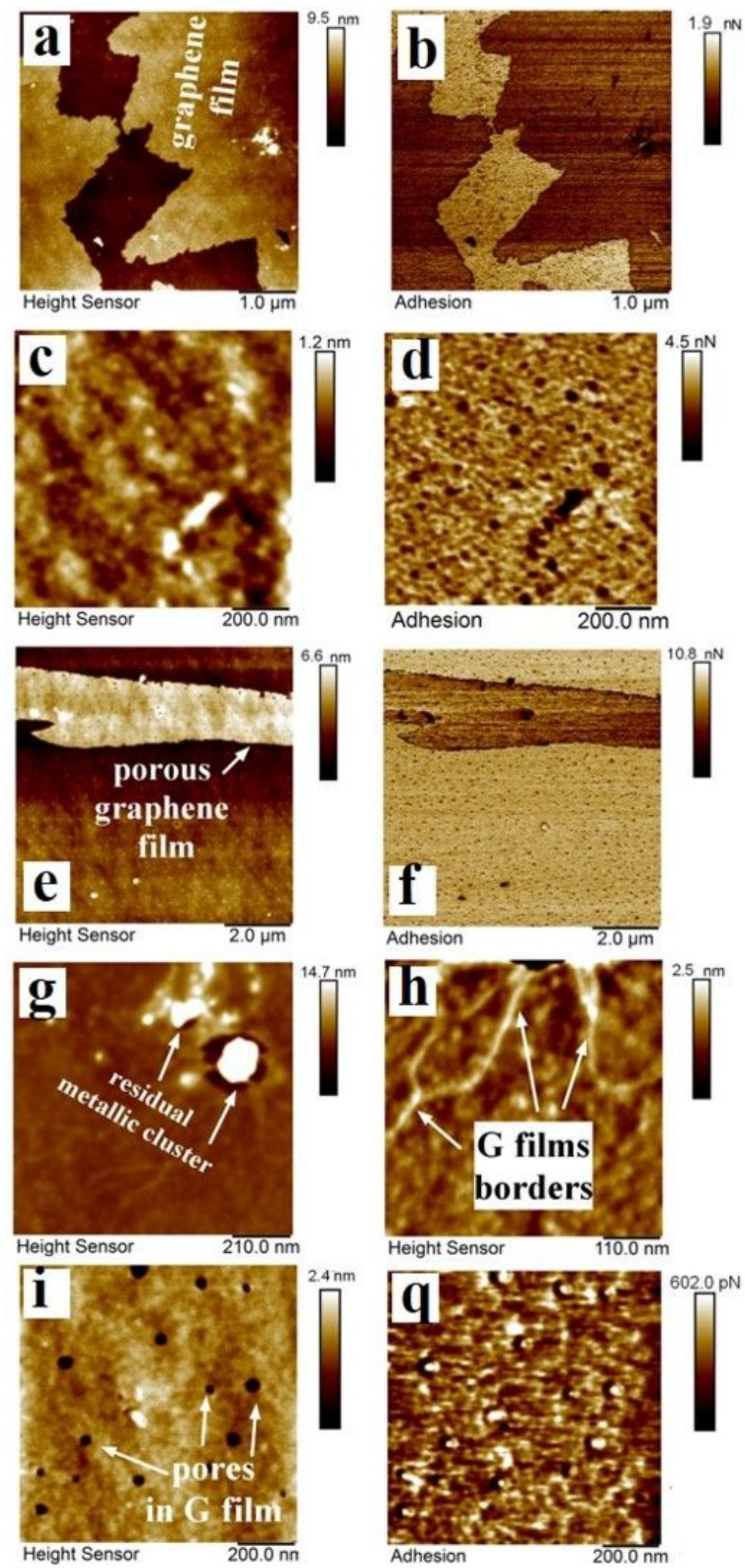

Figure S8. AFM images of G@CuNi etched samples in (a, c, e, g, h and i) topography and (b, $\mathrm{d}, \mathrm{f}$, and q) adhesion force modes 


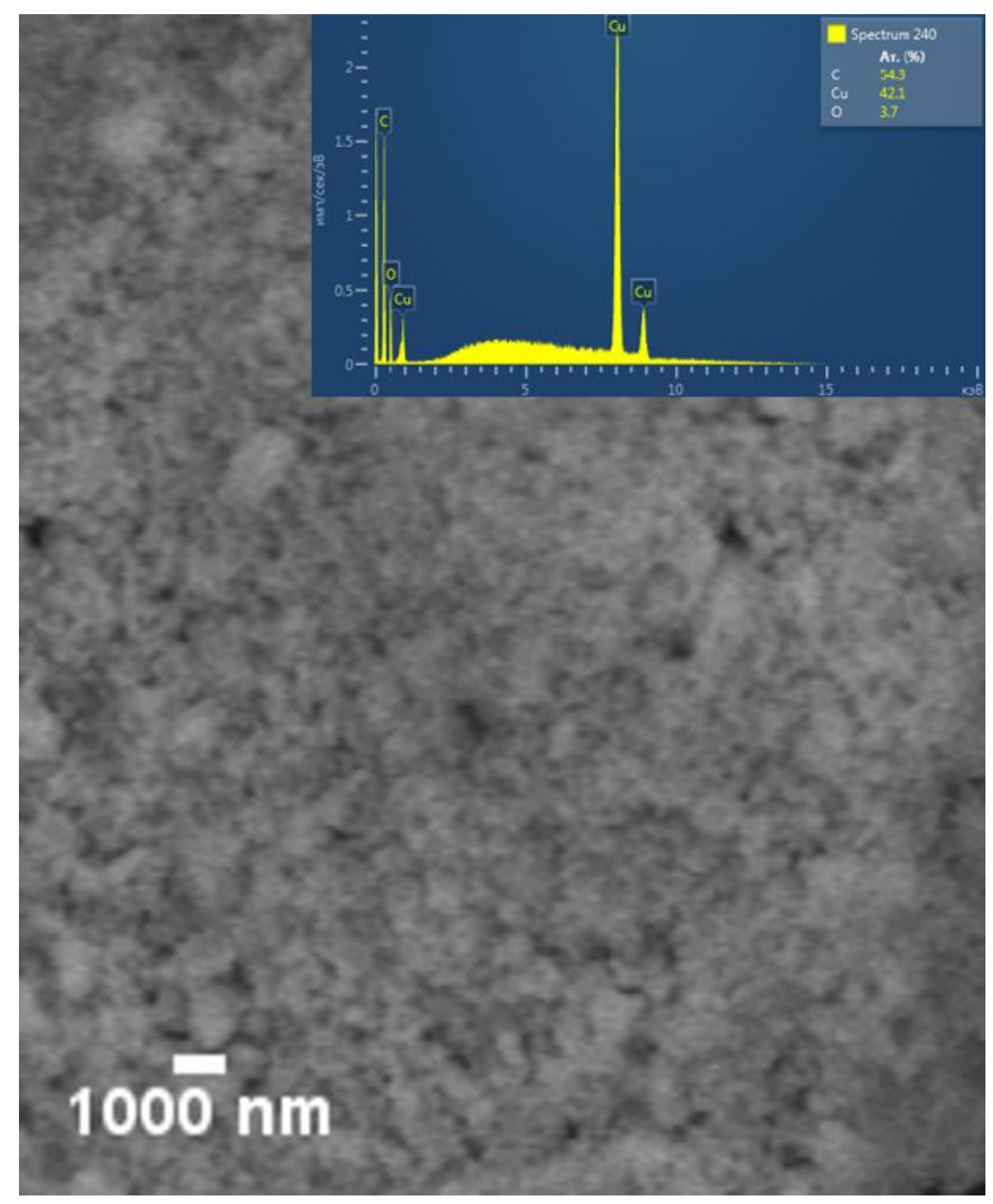

Figure S9. EDX analysis of G $@$ Cu sample 